\title{
Confounding factors complicate conclusions in aly model
}

To the editor-Secondary lymphoid organs are widely understood to play a pivotal role in coordinating adaptive immune responses, though direct demonstrations are few. Most available evidence use as their model the alymphoplastic (aly/aly) mouse which lacks peripheral lymph nodes as well as Peyer's patches ${ }^{1-3}$. Most recently, Lakkis and colleagues demonstrated that splenectomized aly/aly mice are rendered immunologically 'ignorant' of their subsequent cardiac allograft, and aly/aly mice with spleens in place permanently accept skin allografts. The authors conclude that secondary lymphoid organs are essential for mediating rejection. Although they are undoubtedly necessary in certain responses, it is unclear to us that secondary lymphoid organs play an essential role here. Apart from the absence of lymph nodes, aly/aly mice host a variety of other serious immune defects which might confound the authors' findings, including depressed baseline immunoglobulin production and isotype switching, defective T-cell function and faulty homing responses ${ }^{1,5}$.

Lymphotoxin- $\alpha$-deficient $\left(\operatorname{Lta}^{-/-}\right)$and lymphotoxin- $\beta$-receptor-deficient $\left(\mathrm{Ltbr}^{--}\right)$ mice are similarly devoid of lymph nodes and Peyer's patches, but share few other defects additionally associated with aly/aly mice ${ }^{6,7}$. Their T-cell responses indeed appear unperturbed ${ }^{8,9} \cdot$ Lta $^{-/-}$mice reject allogeneic skin grafts in near

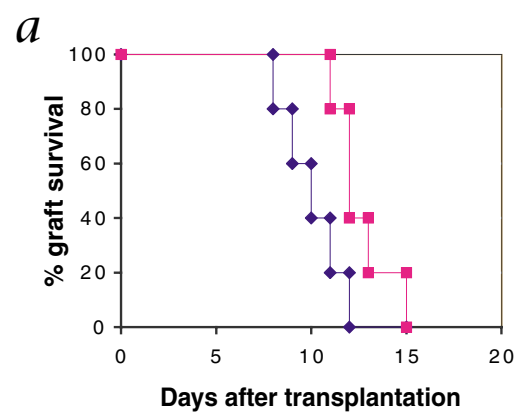

$b$

Fig. 1 Limited role of lymph nodes and spleen in allograft rejection. $\boldsymbol{a}$, Lymph nodes are not essential for the rejection of skin allografts. Full-thickness tail skin was transplanted from 5-8-wk-old $\mathrm{BALB} / \mathrm{C}$ mice to the dorsal flank area of 5-8-wk-old C57BL/6 or B6-Lta ${ }^{-/}$recipients. Rejection was defined as graft necrosis more than $80 \%$. Time course of rejection of allogeneic (BALB/C) skin grafts is near identical in $\operatorname{Lta}^{-/}(\square ; n=5)$ and wild-type $(\forall ; n=5)$ mice. Similar results were also obtained when donor abdominal skin was used. $\boldsymbol{b}$, Lymph nodes and spleen are not essential for the rejection of cardiac allografts. Cardiac allograft transplant was performed using BALB/C donors and $\mathrm{C} 57 \mathrm{BL} / 6(\checkmark ; n=7)$, splenectomized B6-Lta ${ }^{-/-}(\square ; n=5)$ or splenectomized B6 Ltbr $r^{-1}$ $(\boldsymbol{\Lambda} ; n=4)$ recipients. Allograft survival was assessed by palpation. Rejection was defined as cessation of heart beat. Splenectomized B6-Lta ${ }^{-1-}$ and B6-Ltbr $r^{-/-}$recipients show delayed, but fully competent rejection. may be responsible for the immunological 'ignorance' witnessed in aly/aly mice. The salient consideration is that other studies of immune responses studied in aly/aly mice might overestimate the involvement of secondary lymphoid organs $^{1-3}$.

Robert ChIN ${ }^{1}$, PING ZHOU ${ }^{1,2}$,

MARIA-LUisA ALEGRE ${ }^{1,2} \&$

YANG XIN FU ${ }^{1,3}$

${ }^{1}$ Committee on Immunology

${ }^{2}$ Department of Medicine and

${ }^{3}$ Department of Pathology

University of Chicago

Chicago, Illinois, USA

Email: yfu@midway.uchicago.edu

Lakkis replies-Using $\mathrm{Lta}^{-1-}$ and $\mathrm{Ltbr}^{-1}$ mice as models to study the role of secondary lymphoid organs in the alloimmune response, Chin et al. present data that seem to challenge our conclusion that the immune response to a vascularized organ graft can not be initiated in the absence of secondary lymphoid organs ${ }^{4}$, which is based on a systematic study of allograft rejection in the alymphoplastic (aly/aly) mouse. Although the findings of Chin et al. are intriguing, they do not refute our conclusion. We carefully excluded in our study the possibility that failure to mount an alloimmune response in splenectomized aly/aly mice resulted from an intrinsic abnormality in aly/aly T-lymphocyte activation or homing.

First, we showed that aly/aly $\mathrm{T}$ cells mediate allograft rejection in T-cell-deficient hosts that have normal secondary lymphoid organs ${ }^{4}$. We adoptively transferred T-cell-enriched blood leukocytes from naive splenectomized aly/aly mice to recipients of cardiac allografts deficient in T lymphocytes and T-cell receptor- $\beta \delta$. We then observed acute rejection in each recipient albeit at a slower tempo than rejection precipitated by the transfer of naive, wild-type $\mathrm{T}$ cells (median graft survival: 20 and $11 \mathrm{~d}$, respectively). These data clearly indicate that the intrinsic defect in aly/aly $\mathrm{T}$ cells slows the rejection process by a modest degree but does not abolish it.

Second, we showed that transfer of a large number of naive, wild-type $\mathrm{T}$ cells $\left(4-8 \times 10^{7}\right)$ to splenectomized aly/aly mice two days after heart transplantation 